\title{
Treinamento da performance comunicativa em universitários da área da saúde
}

\section{Communicative performance training in university health}

\author{
students
}

\author{
Leticia Corrêa Celeste1, Aveliny Mantovan Lima1', Juliana Moura Alves Seixas', Milena Assis da Silva', Eduardo \\ Magalhães da Silva ${ }^{1}$
}

\section{RESUMO}

Introdução: A expressão do conhecimento é um desafio na vida dos alunos no ensino superior. Objetivo: Avaliar a eficácia da intervenção fonoaudiológica por meio do programa Treinamento da Performance Comunicativa (TAPCo) para universitários. Métodos: Participaram desta pesquisa 26 universitários de ambos os gêneros e de quatro diferentes cursos da área da saúde. A pesquisa foi realizada em seis etapas, a saber: registro audiovisual de uma apresentação oral dos discentes antes do treinamento; treinamento das habilidades comunicativas por meio do programa TAPCo; registro audiovisual após o treinamento; tabulação dos dados obtidos pelos pesquisadores; avaliação randomizada e cega dos 26 discentes, por três fonoaudiólogas não participantes da pesquisa, de forma qualitativa; autoavaliação dos participantes. O TAPCo foi composto por oito sessões de treinamento, que envolveram os seguintes aspectos: vestuário, expressão corporal e facial, fala e voz. Resultados: Houve melhora, com diferença estatisticamente significativa, em todos os aspectos treinados, de acordo com a autoavaliação. Quanto à avaliação qualitativa das juízas, observou-se que a classificação predominante foi boa, em todos os aspectos avaliados. No aspecto vestuário, houve maior presença do indicador indiferente. $\mathrm{O}$ aspecto fala e voz apresentou a maior predominância da qualificação excelente. Conclusão: Tanto as avaliações das juízas, quanto as autoavaliações, mostraram a eficiência do TAPCo para o treinamento de estudantes universitários. Embora a autoavaliação tenha apresentado algumas divergências em relação à avaliação das juízas, esse procedimento se mostrou uma excelente ferramenta de acompanhamento da evolução do TAPCo.

Palavras-chave: Comunicação; Capacitação; Fala; Voz

\begin{abstract}
Introduction: Knowledge expression is a challenge in the lives of students in higher education. Purpose: Evaluation of the efficacy of Speech-Language Pathology intervention in a Communicative Performance Training (TAPCo) program for university students. Methods: Twenty six students of both genres and from four different health courses participated in this study. The research was carried out in six stages: audiovisual record of an oral presentation of the students before the training; training of communicative skills through the TAPCo program; audiovisual recording after training; tabulation of participants by the researchers of this paper; randomized and blinded qualitative evaluation by three Speech-Language Pathologists that did not participate of the research of the 26 students, and; self-assessment performed by the 26 participants. The TAPCo was composed of eight training sessions that involved the following aspects: dress code, body and facial expression, voice and speech. Results: There was significant difference, according to self-assessment, in all trained aspects. Regarding the qualitative classification of judges' evaluations, it was observed that good was predominant in all evaluated aspects. In the dress code aspect was observed that there was a greater presence of the indifferent indicator. The speech and the voice aspects presented the highest predominance of the excellent qualification. Conclusion: Both the evaluations of the judges and the self-assessments showed the efficiency of TAPCo in training university students. Although the self-assessment presented some divergences compared to the judges' evaluation, this procedure proved to be an excellent tool to monitor the evolution of TAPCo.
\end{abstract}

Keywords: Communication; Training; Speech; Voice

Trabalho realizado no Curso de Fonoaudiologia, Universidade de Brasília, Campus Ceilândia - FCE/UnB - Brasília (DF), Brasil.

(1) Curso de Fonoaudiologia, Universidade de Brasília, Campus Ceilândia - FCE/UnB - Brasília (DF), Brasil.

Conflito de interesses: Não

Contribuição dos autores: $L C C$ atuou como pesquisadora principal e orientadora do projeto de pesquisa; $A M L G$ participou da análise e tabulação dos dados; JMAS participou da coleta e análise dos dados; MAS participou da coleta e análise dos dados; EMS participou da análise e discussão dos resultados.

Autor correspondente: Eduardo Magalhães da Silva. E-mail: edumagalhaes@unb.br

Recebido: 28/4/2017; Aceito: 22/11/2017 


\section{INTRODUÇÃO}

No ensino superior, é comum os docentes utilizarem a estratégia didática de apresentação de seminários como método avaliativo, para que ocorra a pesquisa e o compartilhamento de informação entre os alunos ${ }^{(1,2)}$. Desta forma, durante a vida acadêmica, as apresentações em público são habituais. Saber se comunicar e expressar o seu conhecimento exige mais do que o domínio do conteúdo e alcançar êxito em seminários ou palestras é um desafio na vida dos discentes.

A expressividade de um locutor, no momento do discurso, é parte fundamental na contextualização da informação a ser socializada, pois é nesse momento que o pensamento do indivíduo se torna vivo, através da linguagem e da expressão corporal $^{(3)}$. Neste sentido, diversos fatores são essenciais para a boa comunicação, pois esse complexo processo, que se exterioriza pela fala, mas não se reduz a ela, exige a aquisição e o aperfeiçoamento de determinadas habilidades inerentes à competência comunicativa oral ${ }^{(4)}$.

A forma como o conteúdo da mensagem é transmitido engloba diversos aspectos, como a fala ${ }^{(5,6)}$, o corpo ${ }^{(5)}$, a voz ${ }^{(5,6)}$, os gestos, o olhar, a postura corporal, a expressão facial ${ }^{(4,7)} \mathrm{e}$ até mesmo o vestuário ${ }^{(8)}$.

Ressalta-se, entretanto, a dificuldade para unir aspectos tão diversificados da comunicação em um treino de aperfeiçoamento. Sendo assim, na tentativa de minimizar a subjetividade e auxiliar os avaliadores, foi proposta uma avaliação focada em alguns pontos a serem considerados, como voz, linguagem corporal e acurácia, a Public Speaking Rubric ${ }^{(1)}$. Uma vez que o objetivo deste trabalho focou-se nas situações de avaliações de apresentações de trabalhos no ensino superior, aspectos como apresentação do escopo, evidências da pesquisa e defesa argumentativa foram incluídos. Outros estudos que envolveram a avaliação ou treino de aspectos relacionados ao aperfeiçoamento comunicativo mostraram a mesma tendência de variação do meio de avaliação, a partir da demanda da pesquisa ${ }^{(9,10,11,12)}$. A ferramenta de autoavaliação, em especial quanto à expressividade geral, também foi utilizada como recurso para verificação de efetividade de treinamento fonoaudiológico ${ }^{(13)}$.

Apesar dos obstáculos acima listados, o trabalho fonoaudiológico com o aperfeiçoamento dos padrões de fala e voz tem sido foco de alguns estudos na área. Em um estudo quantitativo de intervenção fonoaudiológica, que analisou um grupo de 14 meteorologistas, foi comprovada a eficácia do treinamento fonoaudiológico. Houve melhora da performance comunicativa dos profissionais, com diferença estatisticamente significativa na comparação pré-intervenção e pós-intervenção fonoaudiológica ${ }^{(9)}$. Outro estudo qualitativo, que teve como participantes discentes, mostrou que, após a intervenção fonoaudiológica, os sujeitos apresentaram maior segurança ao falar e melhora na socialização da mensagem ${ }^{(12)}$. Ao estudar o efeito do treinamento fonoaudiológico em telejornalistas, observou-se melhora significativa após intervenção, sob o ponto de vista dos espectadores ${ }^{(10)}$.
No que tange ao ensino superior, foco principal deste estudo, grande parte dos universitários se sente desorientada no momento de uma apresentação oral, situação muitas vezes desencadeada pelo estresse, medo e despreparo ${ }^{(14)}$. Assim, estudos que busquem entender melhor tal processo e verificar quantitativamente a intervenção nesse público são necessários.

Este estudo teve o objetivo de avaliar a eficácia da intervenção fonoaudiológica por meio do programa Treinamento da Performance Comunicativa (TAPCo) para universitários.

\section{MÉTODOS}

Trata-se de um estudo longitudinal prospectivo, aprovado pelo Comitê de Ética em Pesquisa da Faculdade de Ciências da Saúde da Universidade de Brasília, sob o parecer nº 686.976 e protocolo CAAE ${ }^{\circ}$ 21132314.6.0000.0030.

Os participantes foram selecionados dentre os 35 universitários matriculados na disciplina Aprimoramento da Performance Comunicativa (APC). Todos consentiram com a realização da pesquisa e a divulgação dos resultados, por meio da assinatura do Termo de Consentimento Livre e Esclarecido (Resolução ${ }^{\circ}$ 466/2012 e suas complementares), que detalhou os objetivos do estudo e garantiu o sigilo dos dados pessoais.

Participaram deste estudo 26 universitários, entre 20 e 25 anos, vinculados a diferentes cursos da área da saúde de uma universidade pública.

Foram considerados como critérios de inclusão que os universitários estivessem matriculados na disciplina APC e obtido $90 \%$ de frequência às aulas ministradas e, como critérios de exclusão, estarem matriculados no curso de Fonoaudiologia, já terem cursado alguma licenciatura, feito algum curso ou treinamento em oratória ou voz profissional e apresentarem, à época da realização da pesquisa, quaisquer queixas fonoaudiológicas.

A pesquisa foi realizada em seis etapas, a saber:

1. Registro audiovisual (filmadora digital, Sony®, modelo Handycam ${ }^{\circledR}$ DCR-SR45) dos participantes de uma apresentação oral sobre um tema científico do seu interesse, com duração de até três minutos;

2. Treinamento em Performance Comunicativa (TAPCo), realizado por docente fonoaudióloga, com experiência na área. O treinamento foi feito em oito encontros semanais, de 100 minutos cada, sendo seis encontros destinados às atividades práticas (Quadro 1);

3. Registro audiovisual (filmadora digital, Sony®, modelo Handycam ${ }^{\circledR}$ DCR-SR45) dos participantes da mesma apresentação oral sobre um tema científico do seu interesse, com duração de até três minutos;

4. Seleção dos participantes pelos pesquisadores, atendendo aos critérios de inclusão e exclusão. Ressalta-se que os participantes foram selecionados após a realização das atividades, devido à possibilidade de viés do conhecimento dos objetivos; 
5. Avaliação randomizada e cega dos 26 vídeos pré-treinamento e 26 vídeos pós-treinamento, por três profissionais fonoaudiólogas. A avaliação pelas juízas ad hoc foi realizada individualmente e separadamente, em data e horário previamente combinados, sendo os vídeos expostos aleatoriamente, não se distinguindo se haviam sido gravados antes ou após a participação no treinamento. As fonoaudiólogas receberam orientações para a atribuição de notas de zero (discordo totalmente) a 5 (concordo totalmente) ao desempenho do participante, relativo aos aspectos e itens que deveriam ser avaliados: expressão corporal (postura, andar, diversidade gestual, relação gestos/discurso, amplitude gestual), expressão facial (contato de olhos, relação olhar/disfluências, expressividade), fala, voz (articulação, velocidade de fala, pausas, melodia, intensidade de voz, ênfases) e vestuário.

6. Autoavaliação dos estudantes participantes, durante a exposição dos vídeos na disciplina, comparando os vídeos pré-treinamento e pós-treinamento, utilizando a mesma ficha disponibilizada para as juízas.

Para o cálculo do escore total, os escores por item e/ou aspecto foram somados e poderiam variar de zero a 90 , em que zero indicava a não variação do aspecto ou item avaliado e 90 , a utilização de todas as ferramentas e conceitos trabalhados no treinamento, durante a apresentação.

Para responder à pergunta se houve diferenças nos aspectos trabalhados pré-treinamento e pós-treinamento e intracursos, realizou-se análise descritiva e estatística (médias, medianas, desvios padrão, valores mínimo e máximo) dos parâmetros pré-treinamento e pós-treinamento: curso, escores médio e total, por aspecto e item.

Foi utilizado o teste $t$ de Student para dados pareados, com nível de significância de 95\% para cada aspecto e item e, para se comparar quantitativamente a evolução em cada aspecto e/ ou item treinado, foram subtraídas as notas pós-treinamento e pré-treinamento.

Os dados numéricos foram trabalhados em termos de qualidade do treinamento, a partir da avaliação das juízas fonoaudiólogas (Figura 1). Para tanto, foram estabelecidos três intervalos de variação, considerando-se os valores definidos pela média, a variação do desvio padrão e pelos valores mínimo e máximo obtidos nestas avaliações. Os escores mínimo e máximo foram considerados como os limites de variação MÍNIMA e MÁXIMA, respectivamente. O intervalo de variação dos escores definido pela soma e subtração da média com o desvio padrão foi considerado como BOM e os valores obtidos acima e abaixo deste intervalo foram considerados, respectivamente, como EXCELENTES e MODERADOS. A variação pré-treinamento e pós-treinamento com valores iguais a zero receberam a indicação INDIFERENTE.

\section{RESULTADOS}

A amostra deste estudo foi composta por 26 universitários,

Quadro 1. Atividades práticas desenvolvidas durante a disciplina/treinamento

\begin{tabular}{|c|c|c|}
\hline Encontro & Foco & Atividades/treinamento \\
\hline 1 & Postura ereta & $\begin{array}{l}\text { Rotação de ombros para trás e manutenção da postura em pequenos monólogos. } \\
\text { Discussão sobre o tema. } \\
\text { Apresentação oral de tema livre, com foco na manutenção da postura. }\end{array}$ \\
\hline 2 e 3 & $\begin{array}{l}\text { Expressão facial e corporal; manutenção } \\
\text { da postura }\end{array}$ & $\begin{array}{l}\text { Realizar contato visual com interlocutores, movimentos de sobrancelhas, uso do sorriso; } \\
\text { possível utilização do andar durante apresentações, versus movimentação das pernas; } \\
\text { dimensão gestual; diversidade gestual (tipos e variedade de gestos); correlação entre } \\
\text { gestos e conteúdo verbal expressado. } \\
\text { Discussão sobre o tema. } \\
\text { Apresentação oral de tema livre, com foco na manutenção da postura, na expressão } \\
\text { facial e na expressão corporal. }\end{array}$ \\
\hline 4 e 5 & $\begin{array}{l}\text { Articulação, velocidade de fala, pausas, } \\
\text { melodia e ênfase; manutenção da } \\
\text { postura; expressividade facial e corporal }\end{array}$ & $\begin{array}{l}\text { Exercícios de sobrearticulação; diminuição da taxa de articulação, por marcação de tempo } \\
\text { articulatório e uso apropriado de pausas; aumento da variação melódica, por meio de } \\
\text { exercícios utilizando frases previamente demarcadas e "palavras de valor". } \\
\text { Discussão sobre o uso da ênfase e seu valor, com uso em situação simulada de } \\
\text { apresentação oral. } \\
\text { Apresentação oral de tema livre, com foco na manutenção da postura, na expressão facial, } \\
\text { na expressão corporal, articulação, velocidade de fala, pausas e melodia. }\end{array}$ \\
\hline 6 & Vestuário & $\begin{array}{l}\text { Discussão de artigos científicos sobre o tema; estruturar diferentes visuais para diferentes } \\
\text { situações de comunicação, com ênfase na comunicação formal e acadêmica. }\end{array}$ \\
\hline 7 & Integração & $\begin{array}{l}\text { Treinar uma apresentação de curta duração em pequenos grupos; fazer a mesma } \\
\text { apresentação para o grupo maior, tentando integrar o conteúdo do treinamento. }\end{array}$ \\
\hline 8 & Autoavaliação & $\begin{array}{l}\text { Comparar os vídeos realizados antes e após o treinamento, fazendo análise crítica da } \\
\text { diferença observada. }\end{array}$ \\
\hline
\end{tabular}




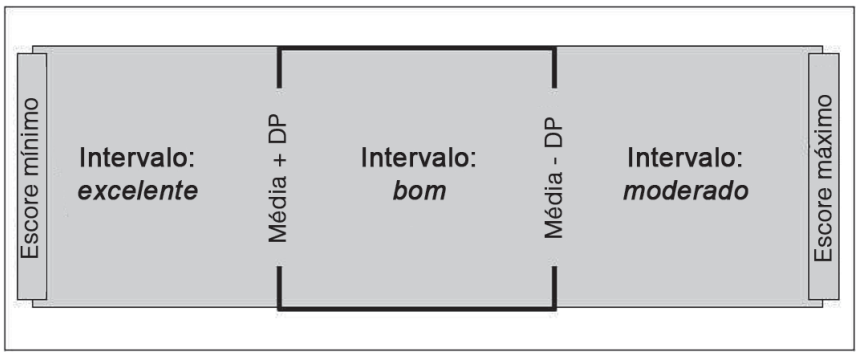

Figura 1. Apresentação gráfica da avaliação de qualidade dos escores pré-treinamento e pós-treinamento, obtidos a partir da avaliação das juízas fonoaudiólogas

$23(88,5 \%)$ do gênero feminino e $3(11,5 \%)$ do gênero masculino, na faixa etária de 20 a 25 anos, matriculados nos cursos de graduação na área da saúde de uma universidade pública federal, sendo 12 estudantes do curso de Enfermagem (46,2\%), 7 de Farmácia (26,9\%), 4 de Fisioterapia (15,4\%) e 3 de Terapia Ocupacional (11,5\%).

Houve evolução positiva de todos os parâmetros trabalhados no treinamento. As médias, medianas e os desvios padrão dos aspectos e itens obtidos nas 52 autoavaliações ( 26 pré-treinamento e 26 pós-treinamento) estão apresentados na Tabela 1.

Quando comparadas as autoavaliações por curso, observou-se diferença estatisticamente significativa em todos os aspectos e itens nos discentes do curso de Enfermagem. O curso de Farmácia somente não apresentou diferença significativa no aspecto vestuário. No curso de Terapia Ocupacional, houve diferença significativa em todos os aspectos, com exceção do item expressividade do aspecto expressão facial e articulação do aspecto fala e voz. No curso de Fisioterapia, houve diferença significativa em todos os itens e aspectos da expressão corporal. No entanto, nos aspectos expressão facial e fala e $v o z$, não houve diferença nos itens relação olhar/disfluências e velocidade de fala e melodia, respectivamente (Tabela 2).

A avaliação das juízas fonoaudiólogas mostrou que houve evolução dos aspectos e itens trabalhados (Tabelas 3 e 4).

$\mathrm{Na}$ avaliação qualitativa, realizada pelas juízas, observou-se que a totalidade dos aspectos recebeu o indicador BOM. No aspecto vestuário, não houve a qualificação MODERADA e, quando comparado aos demais aspectos, ocorreu maior presença do indicador INDIFERENTE. O aspecto fala e voz não apresentou o indicador INDIFERENTE e foi o aspecto em que houve predominância da qualificação EXCELENTE.

\section{DISCUSSÃO}

$\mathrm{O}$ ingresso na universidade traz numerosos desafios, que requerem um novo repertório de estratégias de adaptação e eficiência, para a qual o aluno deve estar preparado. Parte desses alunos apresenta dificuldades para se adaptar a esse ambiente, definindo a necessidade de as universidades prepararem seus estudantes para as novas demandas, evitando experiências estressantes que dificultem o desempenho acadêmico ${ }^{(15)}$. Neste

Tabela 1. Aspectos e itens autoavaliados no pré-treinamento e pós-treinamento, por todos os participantes

\begin{tabular}{|c|c|c|c|c|c|c|c|}
\hline \multirow[t]{2}{*}{ Aspectos e itens } & \multicolumn{3}{|c|}{$\begin{array}{l}\text { Pré-treinamento } \\
\qquad(n=26)\end{array}$} & \multicolumn{3}{|c|}{$\begin{array}{l}\text { Pós-treinamento } \\
\qquad(n=26)\end{array}$} & \multirow[t]{2}{*}{ Valor de $p$} \\
\hline & M & Med & DP & $M$ & Med & DP & \\
\hline Vestuário & 4,2 & 5,0 & 1,2 & 4,9 & 5,0 & 0,4 & $0,002^{*}$ \\
\hline Expressão Corporal & 2,2 & 2,0 & 0,9 & 3,9 & 4,0 & 0,6 & $0,000^{*}$ \\
\hline Postura & 2,4 & 3,0 & 1,2 & 4,3 & 4,0 & 0,5 & $0,000^{*}$ \\
\hline Andar & 2,0 & 2,0 & 1,1 & 4,0 & 4,0 & 1,0 & $0,000^{*}$ \\
\hline Diversidade gestual & 1,9 & 2,0 & 1,1 & 3,5 & 3,0 & 0,8 & $0,000^{*}$ \\
\hline Relação gestos/discurso & 2,3 & 2,0 & 1,3 & 3,8 & 4,0 & 0,8 & $0,000^{*}$ \\
\hline Amplitude gestual & 2,4 & 2,0 & 1,3 & 4,0 & 4,0 & 0,9 & $0,000^{*}$ \\
\hline Expressão Facial & 2,4 & 2,7 & 0,9 & 3,9 & 4,0 & 0,6 & $0,000^{*}$ \\
\hline Contato de olhos & 2,6 & 3,0 & 1,1 & 4,6 & 5,0 & 0,5 & $0,000^{*}$ \\
\hline Relação olhar/disfluências & 2,1 & 2,0 & 1,2 & 3,4 & 4,0 & 1,3 & $0,000^{*}$ \\
\hline Expressividade & 2,6 & 3,0 & 1,1 & 3,7 & 4,0 & 0,9 & $0,000^{*}$ \\
\hline Fala e Voz & 2,8 & 2,8 & 0,9 & 4,1 & 4,2 & 0,5 & $0,000^{*}$ \\
\hline Articulação & 2,9 & 3,0 & 0,9 & 3,9 & 4,0 & 0,6 & $0,000^{*}$ \\
\hline Velocidade de fala & 2,7 & 3,0 & 1,2 & 4,3 & 4,0 & 0,6 & $0,000^{*}$ \\
\hline Pausas & 2,4 & 2,0 & 1,3 & 4,1 & 4,0 & 0,6 & $0,000^{*}$ \\
\hline Melodia & 2,8 & 3,0 & 1,1 & 3,8 & 4,0 & 1,0 & $0,000^{*}$ \\
\hline Intensidade & 3,3 & 4,0 & 1,1 & 4,0 & 4,0 & 0,7 & $0,000^{*}$ \\
\hline Ênfases & 2,4 & 2,0 & 1,1 & 4,4 & 5,0 & 0,8 & $0,000^{*}$ \\
\hline
\end{tabular}

*Valores significativos $(p<0,05)-$ Teste $t$ de Student

Legenda: $\mathrm{M}=$ média; $M e d=$ mediana; $\mathrm{DP}$ = desvio padrão 
Tabela 2. Aspectos e itens autoavaliados no pré-treinamento e pós-treinamento, por curso

\begin{tabular}{|c|c|c|c|c|c|c|c|c|c|c|c|c|c|c|}
\hline \multirow{2}{*}{ Aspectos } & \multirow{2}{*}{ Itens } & \multirow{2}{*}{ Medidas } & \multicolumn{3}{|c|}{$\begin{array}{l}\text { Enfermagem } \\
\qquad(n=12)\end{array}$} & \multicolumn{3}{|c|}{$\begin{array}{l}\text { T. Ocupacional } \\
(n=3)\end{array}$} & \multicolumn{3}{|c|}{$\begin{array}{c}\text { Fisioterapia } \\
\qquad(n=4)\end{array}$} & \multicolumn{3}{|c|}{$\begin{array}{c}\text { Farmácia } \\
\qquad(\mathrm{n}=7)\end{array}$} \\
\hline & & & Pré & Pós & $\begin{array}{l}\text { Valor } \\
\text { de } p\end{array}$ & Pré & Pós & $\begin{array}{l}\text { Valor } \\
\text { de } p\end{array}$ & Pré & Pós & $\begin{array}{l}\text { Valor } \\
\text { de } p\end{array}$ & Pré & Pós & $\begin{array}{l}\text { Valor } \\
\text { de } p^{*}\end{array}$ \\
\hline \multirow{3}{*}{ Vestuário } & & $M$ & 4,3 & 4,8 & & 5,0 & 5,0 & & 3,8 & 4,8 & & 4,1 & 5,0 & \\
\hline & & Med & 4,5 & 5,0 & 0,023 & 5,0 & 5,0 & $0,000^{*}$ & 4,5 & 5,0 & 0,126 & 5,0 & 5,0 & 0,086 \\
\hline & & DP & 1,0 & 0,4 & & 0,0 & 0,0 & & 1,9 & 0,5 & & 1,5 & 0,0 & \\
\hline \multirow{15}{*}{$\begin{array}{l}\text { Expressão } \\
\text { Corporal }\end{array}$} & \multirow{3}{*}{ Postura } & $M$ & 2,1 & 4,2 & & 3,7 & 4,7 & & 2,5 & 4,5 & & 2,3 & 4,3 & \\
\hline & & Med & 2,5 & 4 & 0,023 & 4 & 5 & $0,000^{*}$ & & 4,5 & $0,008^{*}$ & 2 & 4 & $0,003^{\star}$ \\
\hline & & DP & 1,1 & 0,6 & & 0,6 & 0,6 & & 1,3 & 0,6 & & 1,6 & 0,5 & \\
\hline & \multirow{3}{*}{ Andar } & $M$ & 1,9 & 3,8 & & 1,3 & 4 & & 2,3 & 4,3 & & 2,1 & 4,1 & \\
\hline & & Med & 2 & 4 & $0,001^{*}$ & 1 & 4 & $0,047^{*}$ & 2 & 4,5 & $0,008^{*}$ & 2 & 4 & $0,008^{*}$ \\
\hline & & $\mathrm{DP}$ & 1 & 1,3 & & 1,5 & 0 & & 1,5 & 1 & & 1,2 & 0,7 & \\
\hline & \multirow{3}{*}{$\begin{array}{c}\text { Diversidade } \\
\text { Gestual }\end{array}$} & M & 1,8 & 3,3 & & 1,7 & 4,3 & & 2,8 & 3,8 & & 1,7 & 3,4 & \\
\hline & & Med & 2 & 3 & 0,000 & 2 & 4 & $0,028^{*}$ & 2,5 & 3,5 & $0,045^{*}$ & 2 & 4 & $0,018^{*}$ \\
\hline & & $\mathrm{DP}$ & 1 & 0,6 & & 0,6 & 0,6 & & 1,7 & 1 & & 1,3 & 1,1 & \\
\hline & \multirow{3}{*}{$\begin{array}{c}\text { Relação } \\
\text { gestos/ } \\
\text { discurso }\end{array}$} & $M$ & 2,3 & 3,8 & & 1,7 & 4,3 & & 3 & 4 & & 2,3 & 3,7 & \\
\hline & & Med & 2 & 4 & 0,000 & 2 & 4 & $0,007^{*}$ & 3 & 4 & $0,045^{*}$ & 2 & 4 & $0,029^{*}$ \\
\hline & & DP & 1,1 & 0,6 & & 0,6 & 0,6 & & 1,6 & 0,8 & & 1,8 & 1,1 & \\
\hline & \multirow{3}{*}{$\begin{array}{l}\text { Amplitude } \\
\text { Gestual }\end{array}$} & $\mathrm{M}$ & 2,4 & 4,1 & & 2,3 & 4,3 & & 3,8 & 4,5 & & 2 & 3,7 & \\
\hline & & Med & 2 & 4 & 0,000 & 3 & 4 & $0,037^{*}$ & 3,5 & 4,5 & $0,028^{*}$ & 2 & 4 & $0,008^{*}$ \\
\hline & & $\mathrm{DP}$ & 1,2 & 0,8 & & 1,2 & 0,6 & & 1 & 0,6 & & 1,5 & 1,1 & \\
\hline \multirow{9}{*}{$\begin{array}{l}\text { Expressão } \\
\text { Facial }\end{array}$} & \multirow{3}{*}{$\begin{array}{l}\text { Contato de } \\
\text { olhos }\end{array}$} & $\mathrm{M}$ & 2,9 & 4,8 & & 2,3 & 4,3 & & 2,8 & 4,5 & & 2,3 & 4,7 & \\
\hline & & Med & 3 & 5 & 0,000 & 3 & 4 & $0,037^{*}$ & 2,5 & 4,5 & $0,034^{*}$ & 2 & 5 & $0,001^{*}$ \\
\hline & & $\mathrm{DP}$ & 1 & 0,5 & & 1,2 & 0,6 & & 1 & 0,6 & & 1,5 & 0,5 & \\
\hline & \multirow{3}{*}{$\begin{array}{l}\text { Relação olhar/ } \\
\text { disfluências }\end{array}$} & $\mathrm{M}$ & 2,3 & 3,6 & & 2 & 2,3 & & 2,8 & 2,8 & & 1,6 & 4 & \\
\hline & & Med & 2 & 4 & 0,014 & 2 & 3 & 0,333 & 3 & 3 & 0,500 & 2 & 4 & $0,001^{*}$ \\
\hline & & DP & 1,2 & 1,4 & & 0 & 1,2 & & 1,5 & 1,5 & & 1,3 & 1,2 & \\
\hline & \multirow{3}{*}{ Expressividade } & $\mathrm{M}$ & 2,8 & 3,9 & & 2,7 & 3,3 & & 2,8 & 3,8 & & 2 & 3,7 & \\
\hline & & Med & 3 & 4 & 0,007 & 3 & 3 & 0,091 & 3 & 3,5 & $0,045^{*}$ & 2 & 4 & $0,003^{*}$ \\
\hline & & $\mathrm{DP}$ & 1 & 0,8 & & 1,5 & 1,5 & & 0,5 & 1 & & 1,4 & 0,8 & \\
\hline & & $\mathrm{M}$ & 3 & 4,3 & & 2,7 & 3,7 & & 3,5 & 4,5 & & 2,6 & 4,4 & \\
\hline & Articulação & Med & 3 & 4 & $0,003^{*}$ & 3 & 4 & 0,112 & 3,5 & 4,5 & $0,045^{*}$ & 3 & 4 & $0,000^{*}$ \\
\hline & & $\mathrm{DP}$ & 1 & 0,6 & & 0,6 & 0,6 & & 1,3 & 0,6 & & 0,8 & 0,5 & \\
\hline & & $\mathrm{M}$ & 2,7 & 4,3 & & 2,3 & 3,7 & & 3 & 4,3 & & 2,6 & 4 & \\
\hline & Fala & Med & 3 & 4 & $0,000^{*}$ & 2 & 4 & $0,028^{*}$ & 3 & 4,5 & 0,070 & 3 & 4 & $0,012^{*}$ \\
\hline & & $\mathrm{DP}$ & 1,2 & 0,5 & & 0,6 & 0,6 & & 1,8 & 1 & & 1,4 & 0,6 & \\
\hline & & $M$ & 2,5 & 3,9 & & 1,7 & 3,3 & & 3 & 4 & & 2,4 & 3,7 & \\
\hline & Pausas & Med & 2,5 & 4 & $0,000^{*}$ & 2 & 3 & $0,018^{*}$ & 3 & 4 & $0,045^{*}$ & 3 & 4 & $0,005^{\star}$ \\
\hline Fala e Voz & & $\mathrm{DP}$ & 1,3 & 1,4 & & 0,6 & 0,6 & & 1,8 & 1,2 & & 1,3 & 0,5 & \\
\hline rala e voz & & $M$ & 3 & 4,3 & & 1,7 & 3,7 & & 3,3 & 4,3 & & 2,6 & 3,7 & \\
\hline & Melodia & Med & 3 & 4 & $0,000^{*}$ & 2 & 3 & $0,037^{*}$ & 3 & 4,5 & 0,091 & 2 & 4 & $0,033^{*}$ \\
\hline & & DP & 1 & 0,5 & & 0,6 & 1,2 & & 1,3 & 1 & & 1,3 & 0,8 & \\
\hline & & $M$ & 3,4 & 4,6 & & 2,7 & 4,3 & & 3,5 & 4,5 & & 3,3 & 4,3 & \\
\hline & Intensidade & Med & 4 & 5 & $0,001^{*}$ & 2 & 4 & $0,018^{*}$ & 3,5 & 4,5 & $0,046^{*}$ & 4 & 5 & $0,031^{*}$ \\
\hline & & $\mathrm{DP}$ & 1 & 0,5 & & 1,2 & 0,6 & & 1,3 & 0,6 & & 1,4 & 1,5 & \\
\hline & & $\mathrm{M}$ & 2,8 & 3,9 & & 1,7 & 4,7 & & 2,5 & 3,8 & & 2,1 & 3,7 & \\
\hline & Ênfase & Med & 3 & 4 & $0,001^{*}$ & 2 & 5 & $0,000^{*}$ & 2,5 & 3,5 & $0,039^{*}$ & 2 & 4 & $0,000^{*}$ \\
\hline & & $\mathrm{DP}$ & 1,1 & 0,8 & & 0,6 & 0,6 & & 1,3 & 1,0 & & 1,3 & 1,6 & \\
\hline
\end{tabular}

* Valores significativos $(p<0,05)-$ Teste t de Student

Legenda: $\mathrm{M}=$ média; $M e d=$ mediana; $\mathrm{DP}=$ desvio padrão

sentido, um treinamento estruturado, com o objetivo de fornecer estratégias práticas sobre os aspectos do desenvolvimento da performance comunicativa, é uma importante ferramenta para a vida acadêmica e o futuro profissional desses estudantes.
No TAPCo, almejou-se o desenvolvimento de uma produção em que todos os aspectos e os seus respectivos elementos escolhidos para compor o trabalho se exibissem de maneira sinérgica e coerente com a mensagem. Neste sentido, os 
Tabela 3. Aspectos e itens avaliados pelas juízas fonoaudiólogas no pré-treinamento e pós-treinamento, por participante

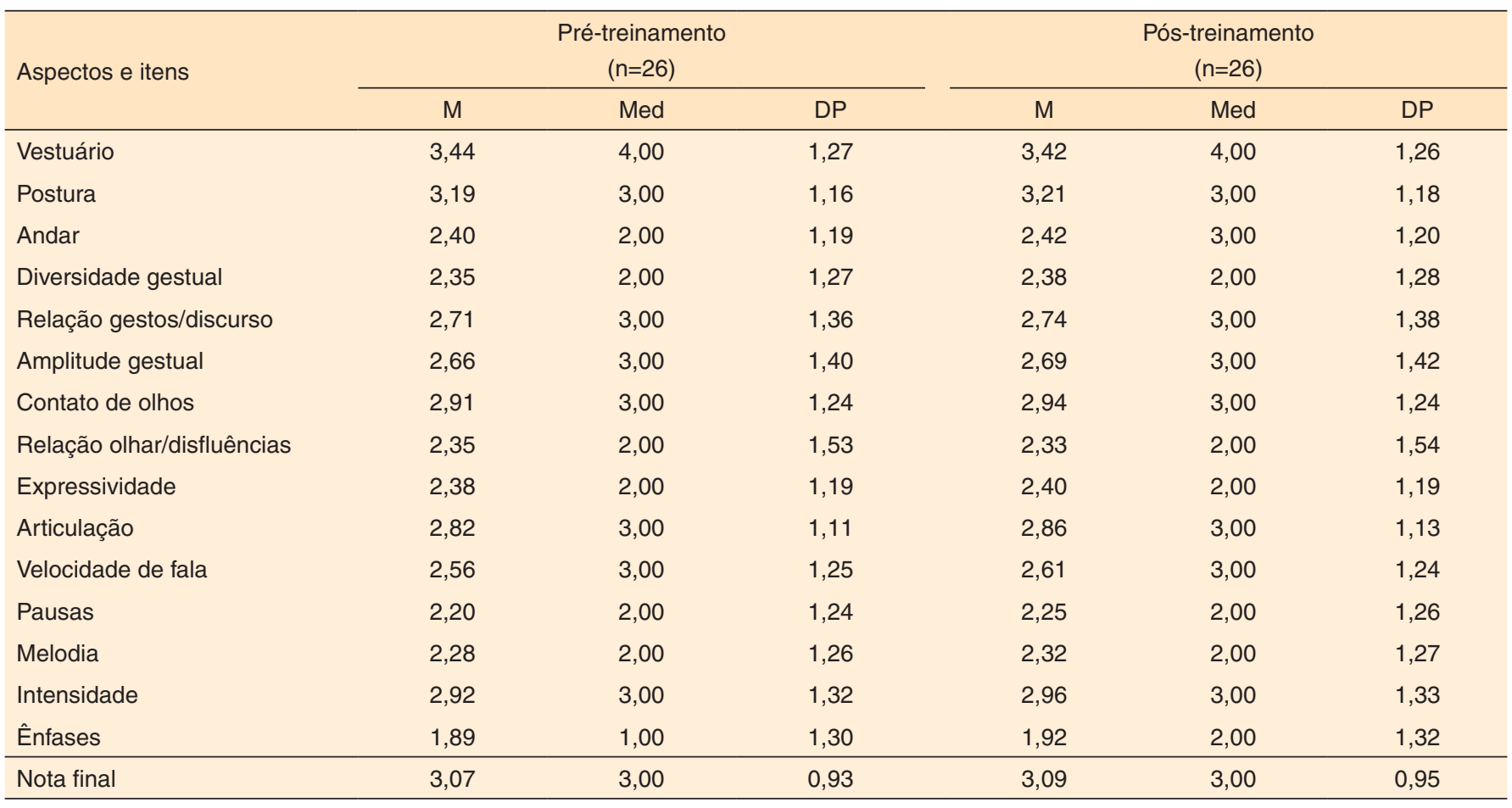

Legenda: $\mathrm{M}$ = média; $M e d=$ mediana; $\mathrm{DP}$ = desvio padrão

Tabela 4. Escores totais dos aspectos avaliados pelas juízas fonoaudiólogas no pré-treinamento e pós-treinamento, por participante

\begin{tabular}{|c|c|c|c|c|c|c|}
\hline \multirow[t]{2}{*}{ Aspectos } & \multicolumn{3}{|c|}{$\begin{array}{l}\text { Pré-treinamento } \\
\qquad(\mathrm{n}=26)\end{array}$} & \multicolumn{3}{|c|}{$\begin{array}{l}\text { Pós-treinamento } \\
\qquad(\mathrm{n}=26)\end{array}$} \\
\hline & $\mathrm{M}$ & Med & $\mathrm{DP}$ & $\mathrm{M}$ & Med & $\mathrm{DP}$ \\
\hline Vestuário & 3,33 & 3,00 & 1,3 & 3,56 & 4,00 & 1,22 \\
\hline Expressão corporal & 2,22 & 2,00 & 0,98 & 3,16 & 3,00 & 1,02 \\
\hline Expressão facial & 2,48 & 2,00 & 0,72 & 2,62 & 3,00 & 0,79 \\
\hline Fala e voz & 2,04 & 2,00 & 0,96 & 2,89 & 3,00 & 1,01 \\
\hline Nota final & 2,83 & 3,00 & 0,87 & 3,35 & 4,00 & 0,94 \\
\hline
\end{tabular}

Legenda: $\mathrm{M}=$ média; $M e d=$ mediana; $\mathrm{DP}$ = desvio padrão

aspectos formais da comunicação foram trabalhados de forma prática e isoladamente, conforme demonstra o Quadro 1, com foco nas apresentações orais na modalidade de conferência.

A elaboração do TAPCo partiu do entendimento de que a comunicação humana inclui os recursos não verbais e verbais da linguagem e, portanto, abriga elementos extralinguísticos, paralinguísticos e linguísticos ${ }^{(16)}$. Desta forma, o elemento extralinguístico vestuário foi tema do sexto encontro (Quadro 1). Esse elemento é entendido como uma atitude comunicativa, que expressa a intenção do falante e que, embora não transmita diretamente uma mensagem, é um aspecto que favorece a confiabilidade no discurso, conforme indicado em estudos ${ }^{(17)}$.

Aos elementos paralinguísticos expressão corporal e expressão facial, foram dedicados três encontros ( 1 a 3 , no Quadro 1). O treino da expressão corporal e da expressão facial auxilia no desempenho das habilidades comunicativas, uma vez que permite a veiculação de emoções, de postura e de gestos que trazem informações comportamentais sobre o falante. Autoras defenderam a necessidade do trabalho de tais aspectos em conjunto com os aspectos linguísticos, para o equilíbrio da comunicação ${ }^{(17)}$.

Por sua vez, então, os elementos linguísticos fala e voz foram trabalhados em dois encontros (4 e 5, do Quadro 1). Relacionados à fala e voz estão os aspectos da produção dos sons da fala e suprassegmentais, referentes à prosódia. $\mathrm{O}$ trabalho dos aspectos prosódicos, tais como pausa, velocidade de fala e ênfase é fundamental para, entre outros aspectos comunicativos, a expressão de atitudes ${ }^{(18)}$, bem como de seleção das informações e contraste de fatos e afetos ${ }^{(19)}$.

É importante ressaltar que, além da estrutura do TAPCo, o número reduzido de participantes permitiu que todos tivessem a oportunidade de experimentar as técnicas com liberdade. Assim, a estrutura, aliada ao número de participantes, resultou em evolução positiva da performance comunicativa dos sujeitos 
deste estudo. Autores recomendaram que o treinamento das habilidades comunicativas seja exclusivamente prático e, para isto, que o grupo seja pequeno, em torno de 15 pessoas $^{(17)}$, o que permite a vivência dos diferentes aspectos envolvidos.

$\mathrm{Na}$ amostra estudada, a maioria $(88,5 \%)$ dos participantes era do gênero feminino. Estudos apontaram que profissões da área da saúde tendem à predominância do gênero feminino ${ }^{(20,21,22)}$, portanto, o maior número de participantes deste gênero era um esperado.

Os resultados referentes à autoavaliação, considerando a comparação pré-TAPCo e pós-TAPCo, demonstraram a evolução positiva para todos os parâmetros trabalhados. Houve exceções para poucos elementos, considerando a análise por curso, tais como vestuário (Farmácia), expressividade (Terapia Ocupacional), relação olhar/disfluências (Fisioterapia), articulação (Terapia Ocupacional), velocidade de fala (Fisioterapia) e melodia (Fisioterapia). Especificamente em relação ao aspecto expressão corporal, foi observada melhora nos resultados após a realização do TAPCo, em todos os participantes, com diferença estatisticamente significativa.

Outros estudos sugeriram que a autoavaliação favorece a autopercepção da evolução apresentada, tanto nas habilidades comunicativas $^{(3)}$, quanto em aspectos específicos, tais como curva melódica, ênfase, uso de pausas, loudness e ressonância ${ }^{(13)}$.

Em relação à avaliação das juízas, propôs-se uma classificação com quatro qualificadores para a evolução dos participantes, na tentativa de observar a gradiência do resultado, ainda que com termos subjetivos. Sendo assim, observou-se que o qualificador intermediário, que indicava evolução BOA, foi predominante em todos os aspectos. Podese afirmar que, de certa forma, esse resultado condiz com o resultado da autoavaliação dos participantes, na medida em que esse último também indicou evolução positiva.

Grande parte dos estudos sobre competência comunicativa é realizada com profissionais que já possuem algum contato com as apresentações em público, como apresentadores ${ }^{(9)} \mathrm{e}$ telejornalistas ${ }^{(10,13)}$, ou com alunos que estão em formação para realizar apresentações em rádio e $\mathrm{TV}^{(12)}$, o que já os predispõe ao uso dessas ferramentas de forma contínua. Assim, ao ingressar no ensino superior são expostos às apresentações de seminários e outras formas de exposição em sala ${ }^{(1,2)}$, o que traz insegurança, uma vez que, grande parte desses universitários não possui um nível de compreensão crítica e intertextual proporcional, resultando em dificuldades, tanto orais, quanto escritas ${ }^{(19)}$.

A participação no processo e a experiência do avaliador são fatores que tornam a avaliação dos aspectos trabalhados intuitiva e subjetiva ${ }^{(23)}$. O examinador treinado valoriza e quantifica aspectos que o participante do processo desconhece ou não valoriza.

Enquanto a autoavaliação traz várias implicações positivas para o indivíduo, pois facilita a autorregulação, fornece uma sensação de autocontinuidade, acelera o processamento de informações relevantes, é uma chave para o estabelecimento de metas, influencia a percepção social, determina a escolha do parceiro, bem como o comportamento em relações pessoais e contribui para a projeção de uma autoimagem consistente e desejável para os outros, podendo este processo se dar por comparação, engajamento, consenso ou experienciação ${ }^{(24)}$. Estudos $^{(25)}$ indicaram que a autoavaliação acadêmica está associada de forma positiva com o desempenho do aluno.

Da mesma forma, o classificador INDIFERENTE (quando a relação pré-TAPCo e pós-TAPCo, foi zero) esteve presente em maior quantidade em vestuário, que foi um dos aspectos observados como não estatisticamente significativo entre os estudantes do curso de Farmácia. Pode-se dizer, portanto, que esses resultados se complementam. Seguindo essa linha de raciocínio, mas como um contraponto, o resultado da avaliação das juízas, em que houve maior predominância do qualificador EXCELENTE foi o do aspecto fala e voz, o que discorda da autoavaliação dos estudantes de Fisioterapia e Terapia Ocupacional para os elementos articulação, velocidade de fala e melodia, que estão contidos no mesmo aspecto.

Estudos mostraram que existe uma diferença entre a percepção dos participantes de treinamentos e os facilitadores desse processo, o que pode ter várias causas hipotéticas, incluindo excesso ou reduzida confiança e ausência de conhecimento. $\mathrm{O}$ tempo de treinamento também pode ser uma causa secundária para os níveis de conhecimento Além disso, fenômenos não qualificados e inconscientes podem desempenhar um papel na autoavaliação imprecisa ${ }^{(26)}$.

Embora no contexto estudado a autoavaliação tenha apresentado algumas divergências em relação à avaliação das juízas, esse procedimento se mostrou uma excelente ferramenta de acompanhamento da evolução do TAPCo. Sabe-se que, na prática fonoaudiológica, nem sempre é possível contar com diferentes especialistas para avaliação do processo de treinamento de um grupo, bem como de seus resultados. Portanto, sugere-se a valorização da autoavaliação, uma vez que se revelou bastante eficaz.

\section{CONCLUSÃO}

O TAPCo é eficaz para o desenvolvimento das habilidades comunicativas de universitários. Tanto a autoavaliação, como a avaliação por juízes, sugerem que a participação no treinamento promove mudanças em todos os aspectos.

\section{REFERÊNCIAS}

1. Schreiber LM, Paul GD, Shibley LR. The development and test of the public speaking competence rubric. Commun Educ. 2012;61(3):205-33. https://doi.org/10.1080/03634523.2012.670709

2. Souza R, Fernandes ACN, Ferreira L. Oficina de expressividade para universitários em situação de apresentação de seminários. Rev Disturb Comum. 2013;25(3):458-76. 
3. Romano CC, Alves LA, Secco IAO, Ricz LNA, Robazzi MLCC. A expressividade do docente universitário durante sua atuação na sala de aula: análise dos recursos verbais utilizados e suas implicações para a enfermagem. Rev Latino-Am Enfermagem. 2011;19(5):118896. https://doi.org/10.1590/S0104-11692011000500017

4. Monteiro C, Viana F, Moreira E, Bastos A. Avaliação da competência comunicativa oral no Ensino Básico: um estudo exploratório. Rev Port Educ. 2013;26(2):111-38. https://doi.org/10.21814/rpe.3248

5. Azevedo LL, Martins PC, Mortimer EF, Quadros AL, Sá EF, Moro L, Pereira RR. Recursos de expressividade usados por uma professora universitária. Rev Disturb Comum. 2014;26(4):777-89.

6. Viola IC, Ghirardi ACAM, Ferreira LP. Expressividade no rádio: a prática fonoaudiológica em questão. Rev Soc Bras Fonoaudiol. 2011;16(1):64-72. https://doi.org/10.1590/S151680342011000100013

7. Araújo MMT, Silva MJP. Estratégias de comunicação utilizadas por profissionais de saúde na atenção à pacientes sob cuidados paliativos. Rev Esc Enferm. 2012;46(3):626-32. https://doi.org/10.1590/S008062342012000300014

8. Petrilli CM, Mack M, Petrilli JJ, Hickner A, Saint S, Chopra V. Understanding the role of physician attire on patient perceptions: a systematic review of the literature: targeting attire to improve likelihood of rapport (TAILOR) investigators. BMJ Open. 2015;5(1):e006578. https://doi.org/10.1136/bmjopen-2014-006578

9. Gampel D. Aspectos de intervenção fonoaudiológica em apresentadores de previsão meteorológica, sem problemas vocais. Rev Disturb Comum. 2006;18(3):355-69.

10. Azevedo JBM, Ferreira LP, Kyrillos L. Julgamento de telespectadores a partir de uma proposta de intervenção fonoaudiológica com telejornalistas. Rev CEFAC. 2009;11(2):281-9. https://doi.org/10.1590/S1516-18462009000200013

11. Borrego MCM, Behlau M. Recursos de ênfase utilizados por indivíduos com e sem treinamento de voz e fala. Rev Soc Bras Fonoaudiol. 2012;17(2):216-24. https://doi.org/10.1590/S151680342012000200019

12. Oliveira GC, Farghali SM, Silva MAA. Fonoaudiologia e formação profissional em rádio e televisão: uma relação produtiva. Rev Disturb Comum. 2013;25(2):293-6.

13. Neiva TMA, Gama ACC, Teixeira LC. Expressividade vocal e corporal para falar bem no telejornalismo: resultados de treinamento. Rev CEFAC. 2016;18(2):498-507. https://doi.org/10.1590/1982021620161829415

14. Osório FL, Crippa JAS, Loureiro SR. Escala para auto-avaliação ao falar em público (SSPS): adaptação transcultural e consistência interna da versão brasileira. Rev Psiq Clin. 2008;35(6):207-11. https://doi.org/10.1590/S0101-60832008000600001
15. Bolsoni-Silva AT, Loureiro SR. Social skills of undergraduates without mental disorders: academic and socio-demographic variables. Psico-USF. 2015;20(3):447-59. https://doi. org/10.1590/1413-82712015200307

16. Laver J. Towards an integrated theory of nonverbal communication. In: Proceedings of the $14^{\text {th }}$ International Congress of Phonetic Sicences - ICPhS99, San Francisco. Berkeley: University of California; 1999. p. 2433-6.

17. Celeste LC, Lima-Gregio AM. Aspectos teóricos para elaboração de programa de treinamento da performance comunicativa. In: Madureira S, organizador. Sonoridades: a expressividade da fala, no canto e na declamação. São Paulo: PUCSP; 2016. p. 111-8.

18. Celeste LC, Reis C. Expressão de certeza e dúvida na gagueira: estudo dos aspectos temporais da fala. Revista CEFAC.2013;15(6):1609-20. https://doi.org/10.1590/S151618462012005000060

19. Kohler K. What is emphasis and how is it coded? In: Proceedings of $3^{\text {rd }}$ International Conference of Speech Prosody, 2006, Dresden, Germany.

20. Teixeira LC, Rodrigues ALV, Santos JN, Cardoso AFR, Gama ACC, Resende LM. Trajetória profissional de egressos em Fonoaudiologia. Rev CEFAC. 2013;15(6):1591-600. https://doi.org/10.1590/S151618462013005000048

21. De Nardi V, Cardoso C, Araujo RPC. Formação acadêmicoprofissional dos docentes fonoaudiólogos do estado da Bahia. Rev CEFAC. 2012;14(6):1122-38. https://doi.org/10.1590/S151618462012005000026

22. Badaró AFV, Guilhem D. Perfil sociodemográfico e profissional de fisioterapeutas e origem das suas concepções sobre ética. Fisioter Mov. 2011;24(3):445-54. https://doi.org/10.1590/S010351502011000300009

23. Silva RSA, Simões-Zenari M, Nemr NK. Impacto do treinamento auditivo na avaliação perceptivo-auditiva da voz realizada por estudantes de Fonoaudiologia. J Soc Bras Fonoaudiol. 2012;24(1):19-25. https://doi.org/10.1590/S217964912012000100005

24. Sedikides C. Assessment, enhancement, and verification determinants of the self-evaluation process. J Pers Soc Psychol. 1993;65(2):317-338. https://doi.org/10.1037/0022-3514.65.2.317

25. DuPaul GJ, Pinho TD, Pollack BL, Gormley MJ, Laracy SD. First-year college students with ADHD and/or LD: differences in engagement, positive core self-evaluation, school preparation, and college expectations. J Learn Disabil. 2017;50(3):238-51. https:// doi.org/10.1177/0022219415617164

26. Quick JA, Kudav V, Doty J, Crane M, Bukoski AD, Bennett BJ, Barnes SL. Surgical resident technical skill self-evaluation: increased precision with training progression. J Surg Res. 218:144-9. https:// doi.org/10.1016/j.jss.2017.05.070 